\title{
Highly Efficient, Environment-Friendly, One-Pot Synthesis of 2-Substituted 4-Formylimidazoles from 4-Acylaminoisoxazoles
}

\author{
Feng Gao*a \\ Xin-Chuan Tian ${ }^{a}$ \\ Xiao-Xia Qu ${ }^{a}$ \\ Dan Wanga \\ Dong $\mathrm{Pu}^{\mathrm{b}}$ \\ a Department of Chinese Traditional Herbal, Agronomy College, \\ Sichuan Agricultural University, No 211, Huimin Road, \\ Wenjing Region, Chengdu 611130, P. R. of China \\ gaofeng@sicau.edu.cn \\ b State Key Laboratory of Biotherapy, Sichuan University, \\ Chengdu 610041, P. R. of China
}<smiles>[R]C(=O)Nc1cnoc1</smiles>

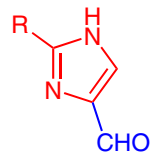

12 examples up to $93 \%$ yield $\quad \mathrm{CHO}$

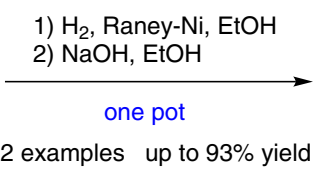

Received: 27.07.2014

Accepted after revision: 01.09.2014

Published online: 30.09 .2014

DOI: 10.1055/s-0034-1379201; Art ID: ss-2014-h0468-op

Abstract A highly efficient and environment-friendly one-pot synthesis of 2-substituted 4-formylimidazoles was accomplished. Raney nickel catalyzed hydrogenation of 4-acylaminoisoxazoles in ethanol, followed by sodium hydroxide promoted recyclization of the ring-opened intermediates, afforded the functionalized imidazoles.

Key words imidazoles, isoxazoles, one-pot synthesis, hydrogenation, environment-friendly

Imidazoles have always been a hot topic for research in organic and pharmaceutical chemistry because of their broad-spectrum bioactivities and synthetic applications. ${ }^{1}$ Among the drugs containing an imidazole ring, antifungal drugs such as miconazole and ketoconazole, which block the biosynthetic pathway of ergosterol by the selective inhibition of fungal cytochrome P450, are well known. ${ }^{2}$ Some alkaloids also contain an imidazole ring and exhibit many prominent bioactivities such as antimicrobial activity and cytotoxicity. ${ }^{1,3}$ Recently, other diverse, interesting bioactivities of imidazoles, such as antikinase IspE activity, ${ }^{4}$ tubulin depolymerization inhibition ${ }^{5}$ and anti-cyclooxygenase activity, ${ }^{6}$ have been demonstrated. Moreover, imidazoles are important precursors of ligands and polymers. Imidazoles can coordinate to various metal ions, forming various metal-organic polymers with complex structures and different functions. ${ }^{7}$ Because of the significant bioactivities and synthetic applications of imidazoles, methods for their synthesis have been extensively studied, including the classical Debus-Radziszewski imidazole synthesis from a diketone, an aldehyde and ammonia. ${ }^{8}$ Several new methods for the synthesis of novel multisubstituted imidazoles have been reported recently. ${ }^{9}$
2-Substituted 4-formylimidazoles, important intermediates in organic and inorganic chemistry, have been used in the synthesis of bioactive compounds, ${ }^{7 \mathrm{~d}, 10}$ metal-organic frameworks ${ }^{7 a}$ and some important ligands. ${ }^{11}$ Because of the basicity of imidazoles, Lewis acid catalyzed Friedel-Crafts reactions are not possible. Therefore, to synthesize functionalized imidazoles, substituents should be introduced prior to the imidazole ring formation. ${ }^{12}$ To the best of our knowledge, mainly two synthetic methods have been reported for the synthesis of 2-substituted 4-formylimidazoles (Scheme 1): a) (Hydroxymethyl)imidazoles have been synthesized by the cyclization of imino ethers with dihydroxyacetone, and subsequent oxidation afforded the corresponding aldehydes; ${ }^{10 \mathrm{~b}}$ b) 2-Aminoimidazoles have been synthesized by the cyclization reaction of 3-bromo-1,1-dimethoxypropan-2-one with guanidine, and subsequent multistep syntheses afforded 2-substituted 4-formylimidazoles. ${ }^{13}$ However, the methods suffer from disadvantages such as heavy metal oxidation and multistep procedures. In 1987, Reiter reported the synthesis of 1-substituted 4(5)acylimidazoles from 4-aminoisoxazoles by palladium on carbon catalyzed hydrogenation, followed by base-promoted cyclization; however, only one example of a formylimidazole in a low yield was reported. ${ }^{14}$ Furthermore, in Reiter's protocol, haloaryl-substituted imidazole-4-carbaldehydes could not be obtained and the reactions occurred under high pressure. Several examples of different substituted acylimidazoles have also been synthesized following Reiter's method..$^{15}$ In our ongoing study of paclitaxel mimics as anticancer drugs, ${ }^{16}$ various 2 -substituted 4 -formylimidazoles were needed as synthetic intermediates. Based on Reiter's result, we have developed a highly efficient, 'onepot' method for the synthesis of 2-substituted 4-formylimidazoles, in which 4-acylaminoisoxazoles, as the starting materials, are subjected to Raney nickel catalyzed hydrogenation, followed by sodium hydroxide promoted recyclization (Scheme 1). 


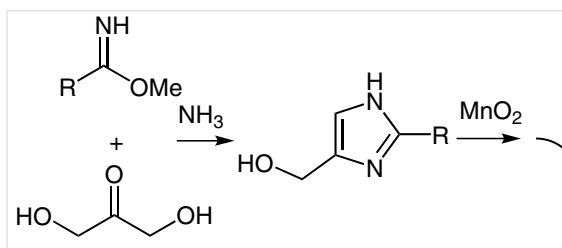

a) previous work<smiles>COC(OC)C(=O)CBr</smiles><smiles>COC(OC)c1c[nH]c(N)n1</smiles>

b) previous work

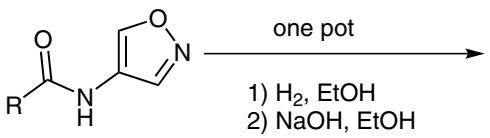

c) this work

Scheme 1 Strategies for the synthesis of 2-substituted 4-formylimidazoles

4-Chloro- $N$-(isoxazol-4-yl)benzamide (1) was used as the starting material to optimize the reaction conditions for the synthesis of 2-(4-chlorophenyl)-4-formyl-1H-imidazole (1a) (Table 1). First, ethanol was used as the solvent for the palladium on carbon catalyzed hydrogenation of 1; LC-MS analysis showed a low yield of intermediate $\mathrm{N}$-(1-amino-3oxoprop-1-en-2-yl)-4-chlorobenzamide (2). Further, the yield of $\mathbf{2}$ decreased with increasing hydrogenation time from 6 to 10 hours (Table 1, entries 1-3). When ethanol was replaced with water, a slight increase in the yield of intermediate 2 was observed (Table 1, entry 4). A careful analysis of the LC-MS results of the hydrogenation indicated that the ring-opening reaction of isoxazole $\mathbf{1}$ was accompanied by the hydrodechlorination of the aryl substituent, thus affording a byproduct, $N$-(1-amino-3-oxoprop-1-en-2yl)benzamide. Therefore, the catalyst for the hydrogenation reaction was changed from palladium on carbon to Raney nickel, which successfully avoided the dehalogenation reaction, thus affording the key intermediate 2 in 92\% yield. Under the alkaline conditions ( $\mathrm{NaOH}, \mathrm{EtOH})$, the primary amino group of compound $\mathbf{2}$ attacks the carbonyl of the amide group to afford the corresponding Schiff base, thus giving the imidazole ring after dehydration. Next, sodium hydroxide ( 2 equiv) was added directly into the reaction mixture after removal of the hydrogenation catalyst by filtration. LC-MS analysis showed a complete conversion of intermediate 2 after 4 hours at $45{ }^{\circ} \mathrm{C}$ to afford 2-(4-chlorophenyl)4-formyl-1 $\mathrm{H}$-imidazole (1a) in $88 \%$ isolated yield (Table 1 , entry 5). After the successful synthesis of the desired com- pound in a high yield, the reaction conditions were further optimized; however, the yield significantly decreased when the temperature of the recyclization step was decreased from $45^{\circ} \mathrm{C}$ to $25^{\circ} \mathrm{C}$, when water was used as the solvent, and when a weaker base $\left(\mathrm{Na}_{2} \mathrm{CO}_{3}\right)$ was used in ethanol (Table 1, entries 6-8).

Table 1 Optimization of the Reaction Conditions

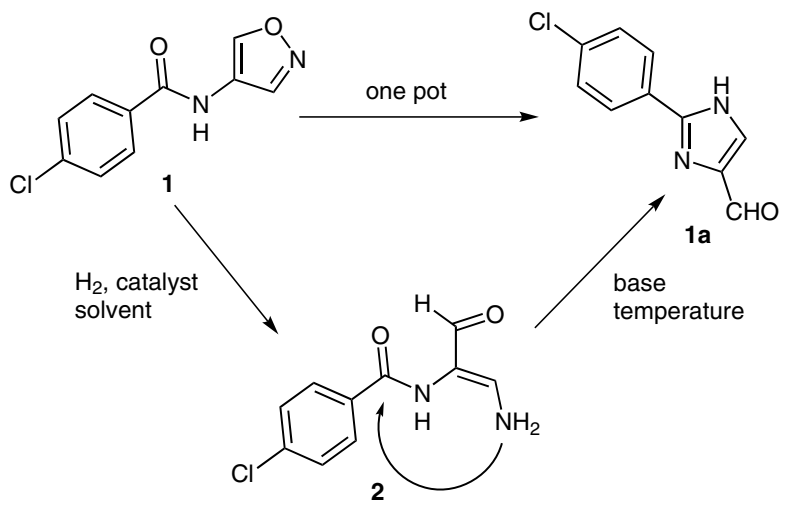

\begin{tabular}{|c|c|c|c|c|c|}
\hline Entry ${ }^{a}$ & Solvent, base ${ }^{b}$ & $\operatorname{Temp}\left({ }^{\circ} \mathrm{C}\right)$ & Time $^{c}(\mathrm{~h})$ & $\begin{array}{l}\text { Yield }^{d}(\%) \\
\text { of } \mathbf{2}\end{array}$ & $\begin{array}{l}\text { Yield }^{d}(\%) \\
\text { of } \mathbf{1 a}\end{array}$ \\
\hline 1 & $\mathrm{EtOH}, \mathrm{Na}_{2} \mathrm{CO}_{3}$ & 45 & 6,6 & 25 & 5 \\
\hline 2 & $\mathrm{EtOH}, \mathrm{NaOH}$ & 45 & 8,6 & 20 & 10 \\
\hline 3 & $\mathrm{EtOH}, \mathrm{NaOH}$ & 45 & 10,6 & 16 & 8 \\
\hline 4 & $\mathrm{H}_{2} \mathrm{O}, \mathrm{NaOH}$ & 45 & 6,6 & 45 & 32 \\
\hline 5 & $\mathrm{EtOH}, \mathrm{NaOH}$ & 45 & 6,4 & 92 & $88^{e}$ \\
\hline 6 & $\mathrm{EtOH}, \mathrm{NaOH}$ & 25 & 6,4 & 92 & 45 \\
\hline 7 & $\mathrm{H}_{2} \mathrm{O}, \mathrm{NaOH}$ & 25 & 6,8 & 15 & 12 \\
\hline 8 & $\mathrm{EtOH}, \mathrm{Na}_{2} \mathrm{CO}_{3}$ & 45 & 6,8 & 92 & 35 \\
\hline \multicolumn{6}{|c|}{$\begin{array}{l}\text { a Entries } 1-4: 5 \% \mathrm{Pd} / \mathrm{C} \text { was used as the hydrogenation catalyst; entries } 5-8 \text { : } \\
5 \% \text { Raney Ni was used as the hydrogenation catalyst. } \\
\text { b Base ( } 2 \text { equiv) was used. } \\
\text { c The first time refers to the hydrogenation reaction (step } 1 \text { ), and the sec- } \\
\text { ond time to the recyclization reaction (step } 2 \text { ). } \\
{ }^{d} \text { LC-MS yields. } \\
\text { e Isolated yield. }\end{array}$} \\
\hline
\end{tabular}

Next, we investigated the substrate scope of different Nsubstituted 4-acylaminoisoxazoles under the optimized reaction conditions $\left[\mathrm{H}_{2}, 5 \%\right.$ Raney $\mathrm{Ni}, \mathrm{NaOH}$ (2 equiv), EtOH] (Table 2). The desired product could be obtained in high yield when the substituent was an aromatic group (Table 2, entries 1-11), while the yield significantly decreased when the substituent was an alkyl group (Table 2, entry 12). These results can be attributed to the fact that an aromatic group extends the $\pi$-conjugation of the system, thus stabilizing the corresponding intermediate and product. Moreover, the electronic nature of the substituents on the benzene ring also slightly affected the yield: the yields were slightly decreased with electron-withdrawing substituents (Table 2, entries 8-10) compared to electron-donating substituents (Table 2, entries 4 and 5). Further investigations 
revealed no significant difference occurring during the hydrogenation reaction, while the yields of the reactions of the substrates with electron-withdrawing substituents at the para position of the aromatic ring decreased after the second step, recyclization, under alkaline conditions. The electron-withdrawing effect may increase the electropositivity of the carbonyl carbon on the amide group, thus facilitating the formation of a Schiff base. This indicates that the rate-limiting step of the recyclization reaction is the dehydration of the 2,3-dihydroimidazole. The electron-donating substituents may facilitate the dehydration reaction, thus affording the final products in high yields.

Table 2 One-Pot Synthesis of 2-Substituted 4-Formylimidazoles from 4-Acylaminoisoxazoles ${ }^{a}$<smiles>O=C(Nc1cnoc1)c1ccc(Cl)cc1</smiles>

1

Product

2<smiles>O=C(Nc1cnoc1)c1ccccc1Cl</smiles>

2

3<smiles>O=C(Nc1cnoc1)c1cccc(Cl)c1</smiles>

3<smiles>O=Cc1c[nH]c(-c2ccc(Cl)cc2)n1</smiles>

1a (88\%)<smiles>O=Cc1c[nH]c(-c2ccccc2Cl)n1</smiles>

$2 a(85 \%)$<smiles>O=Cc1c[nH]c(-c2cccc(Cl)c2)n1</smiles>

3a $(87 \%)$<smiles>COc1ccc(C(=O)Nc2cnoc2)cc1</smiles>

4<smiles>COc1ccc(-c2nc(C=O)c[nH]2)cc1</smiles>

4a (90\%)

5<smiles>COc1cccc(C(=O)Nc2cnoc2)c1</smiles>

5<smiles>COc1cccc(-c2nc(C=O)c[nH]2)c1</smiles>

5a $(93 \%)$<smiles>O=C(Nc1cnoc1)c1ccc(OCC(F)(F)F)cc1</smiles>

6<smiles>CCOc1ccc(-c2nc(C=O)c[nH]2)cc1</smiles>

6a (85\%)

\section{Table 2 (continued)}<smiles>O=C(Nc1cnoc1)c1ccc(C(F)(F)F)cc1</smiles>

7

$7 a(84 \%)$

Product<smiles>O=Cc1c[nH]c(-c2ccc(C(F)(F)F)cc2)n1</smiles><smiles>O=C(Nc1cnoc1)c1cc(F)cc(F)c1</smiles>

8<smiles>O=Cc1c[nH]c(-c2cc(F)cc(F)c2)n1</smiles>

$8 a(78 \%)$<smiles>O=C(Nc1cnoc1)c1cc(F)ccc1F</smiles>

9

10<smiles>O=C(Nc1cnoc1)c1cccc(F)c1F</smiles>

10<smiles>O=Cc1c[nH]c(-c2cc(F)ccc2F)n1</smiles>

9a $(79 \%)$<smiles>O=Cc1c[nH]c(-c2cccc(F)c2F)n1</smiles>

$10 a(80 \%)$

11<smiles>O=C(Nc1cnoc1)c1cccnc1</smiles><smiles>O=Cc1c[nH]c(-c2cccnc2)n1</smiles>

11

$11 a(93 \%)$

12<smiles>CC(C)CC(=O)Nc1cnoc1</smiles>

12<smiles>CC(C)Cc1nc(C=O)c[nH]1</smiles>

$12 a(65 \%)$

a Reaction conditions: $\mathrm{H}_{2}, 5 \%$ Raney Ni, $\mathrm{NaOH}$ (2 equiv), EtOH.

Finally, we scaled up the reaction with $\mathrm{N}$-(isoxazol-4yl)-4-methoxybenzamide (4) to a $100-\mathrm{mmol}$ scale. The desired product, 4-formyl-2-(4-methoxyphenyl)- $1 \mathrm{H}$-imidazole (4a), was obtained in $85 \%$ yield (Scheme 2 ).

In summary, we have developed a novel, one-pot method for the synthesis of 2-substituted 4-formylimidazoles. In this method, 4-acylaminoisoxazoles are used as the starting materials. Raney nickel catalyzed hydrogenation of the 4acylaminoisoxazoles in ethanol affords the ring-opened intermediates. Sodium hydroxide promoted, Schiff base cyclization of the intermediate in the same pot results in an imidazole ring after dehydration, thus finally affording the 
<smiles>COc1ccc(C(=O)Nc2cnoc2)cc1</smiles>

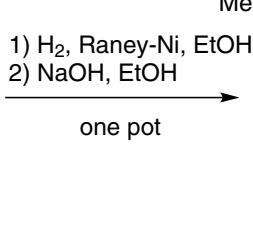

$\mathrm{MeO}$<smiles>O=Cc1c[nH]c(-c2ccc(F)cc2)n1</smiles>
4a $17.1 \mathrm{~g}(85 \%)$

Scheme 2 Scaled-up synthesis of 4-formyl-2-(4-methoxyphenyl)-1Himidazole (4a)

2-substituted 4-formylimidazole in high yield. Because this method is highly efficient and eco-friendly, it can be used for the rapid synthesis of 4-formylimidazole derivatives with different aromatic substituents at the $\mathrm{C}-2$ position of the imidazole ring. Further studies in this direction are underway in our laboratory.

${ }^{1} \mathrm{H}$ and ${ }^{13} \mathrm{C}$ NMR spectra were obtained in $\mathrm{CDCl}_{3}, \mathrm{CD}_{3} \mathrm{OD}$ or DMSO- $d_{6}$ with TMS as the internal standard on a Varian Unity Inova 400/54 spectrometer. Mass spectra were obtained on a VG Auto Spec 3000 or a Finnigan MAT 90 instrument. IR spectra were obtained with $\mathrm{KBr}$ plates using a Perkin-Elmer Spectrum 1600 Series spectrometer. Melting points were determined on a Thomas Hoover Unimelt melting point apparatus and are uncorrected. Silica gel $\mathrm{H}$ (Qingdao Sea Chemical Factory, Qingdao, China) was used for column chromatography. Spots on TLC (silica gel G) were detected by UV light. Commercially available reagents and solvents were directly used without further purification.

\section{2-Substituted 4-Formylimidazoles; General Procedure}

A 4-(acylamino)isoxazole $(0.1 \mathrm{mmol})$ was hydrogenated under hydrogen (balloon) over Raney Ni (5\% by weight) in EtOH (ca. $10 \mathrm{~mL}$ per $\mathrm{mmol}$ of isoxazole). The reaction was usually finished after $1 \mathrm{~h}$, as determined by TLC (hexane-EtOAc, 2:1); then, the catalyst was removed by filtration and washed with EtOH. The combined EtOH layer containing the intermediate $\mathrm{N}$-(1-amino-3-oxoprop-1-en-2-yl)amide was treated with $\mathrm{NaOH}$ ( 2 equiv) at $45^{\circ} \mathrm{C}$ for $4 \mathrm{~h}$. Then, the solvent was removed under reduced pressure and the residue was purified by chromatography on silica gel (hexane-EtOAc, ca. $4: 1, v / v$ ) to give the desired product.

\section{2-(4-Chlorophenyl)-4-formyl-1H-imidazole (1a) ${ }^{10 a}$}

White amorphous powder; yield: $18.1 \mathrm{mg}(88 \%) ; \mathrm{mp} 142-143^{\circ} \mathrm{C}$.

${ }^{1} \mathrm{H}$ NMR (400 MHz, DMSO- $d_{6}$ ): $\delta=9.79(\mathrm{~s}, 1 \mathrm{H}), 8.00(\mathrm{~s}, 1 \mathrm{H}), 7.96$ (d, $J=8.4 \mathrm{~Hz}, 2 \mathrm{H}), 7.51(\mathrm{~d}, J=8.4 \mathrm{~Hz}, 2 \mathrm{H})$.

${ }^{13} \mathrm{C}$ NMR $\left(100 \mathrm{MHz}\right.$, DMSO- $\left.d_{6}\right): \delta=183.8,150.7,140.2,137.0,130.2$, 129.1, 128.9, 127.9.

HRMS (ESI): $m / z\left[\mathrm{M}+\mathrm{H}^{+}\right]$calcd for $\mathrm{C}_{10} \mathrm{H}_{8} \mathrm{ClN}_{2} \mathrm{O}$ : 207.0325; found: 207.0339 .

\section{2-(2-Chlorophenyl)-4-formyl-1H-imidazole (2a) ${ }^{10 a}$}

White amorphous powder; yield: $17.5 \mathrm{mg}(85 \%) ; \mathrm{mp} 145-147^{\circ} \mathrm{C}$.

${ }^{1} \mathrm{H}$ NMR (400 MHz, $\left.\mathrm{CD}_{3} \mathrm{OD}\right): \delta=9.83(\mathrm{~s}, 1 \mathrm{H}), 8.05$ (s, $1 \mathrm{H}$ ), 7.74 (s, 1 $\mathrm{H}), 7.57$ (s, $1 \mathrm{H}), 7.48$ (br s, $2 \mathrm{H}$ ).

${ }^{13} \mathrm{C}$ NMR (100 MHz, $\left.\mathrm{CD}_{3} \mathrm{OD}\right): \delta=184.5,148.9,140.5,133.8,132.8$, $132.5,132.0,131.4,130.1,128.4$

HRMS (ESI): $m / z\left[\mathrm{M}+\mathrm{H}^{+}\right]$calcd for $\mathrm{C}_{10} \mathrm{H}_{8} \mathrm{ClN}_{2} \mathrm{O}$ : 207.0325; found: 207.0336 .
2-(3-Chlorophenyl)-4-formyl-1H-imidazole (3a) $)^{10 a}$

White amorphous powder; yield: $17.9 \mathrm{mg}$ (87\%); mp 152-153 ${ }^{\circ} \mathrm{C}$.

${ }^{1} \mathrm{H}$ NMR $\left(400 \mathrm{MHz}\right.$, DMSO- $\left.d_{6}\right): \delta=13.58(\mathrm{~s}, 1 \mathrm{H}), 9.78(\mathrm{~s}, 1 \mathrm{H}), 8.15(\mathrm{~s}$, $1 \mathrm{H}), 8.12(\mathrm{~s}, 1 \mathrm{H}), 8.03(\mathrm{~d}, J=6.8 \mathrm{~Hz}, 1 \mathrm{H}), 7.52(\mathrm{~m}, 2 \mathrm{H})$.

${ }^{13} \mathrm{C}$ NMR $\left(100 \mathrm{MHz}\right.$, DMSO- $\left.d_{6}\right): \delta=183.2,147.5,133.6,131.4,130.8$, $129.9,129.1,128.1,125.4,124.3$.

HRMS (ESI): $m / z\left[\mathrm{M}+\mathrm{H}^{+}\right]$calcd for $\mathrm{C}_{10} \mathrm{H}_{8} \mathrm{ClN}_{2} \mathrm{O}$ : 207.0325; found: 207.0331.

\section{4-Formyl-2-(4-methoxyphenyl)-1H-imidazole (4a) $)^{10 a}$}

White solid; yield: $18.2 \mathrm{mg}$ (90\%); $\mathrm{mp} 167-168^{\circ} \mathrm{C}$.

${ }^{1} \mathrm{H}$ NMR (400 MHz, $\left.\mathrm{CD}_{3} \mathrm{OD}\right): \delta=9.74(\mathrm{~s}, 1 \mathrm{H}), 7.94(\mathrm{~s}, 1 \mathrm{H}), 7.90(\mathrm{~d}, J=$ $8.8 \mathrm{~Hz}, 2 \mathrm{H}), 7.03$ (t, $J=8.8 \mathrm{~Hz}, 2 \mathrm{H}), 3.85(\mathrm{~s}, 3 \mathrm{H})$.

${ }^{13} \mathrm{C}$ NMR $\left(100 \mathrm{MHz}, \mathrm{CD}_{3} \mathrm{OD}\right): \delta=183.2,162.7,152.2,129.0,128.0$, 122.6, 115.4, 115.2, 55.9.

HRMS (ESI): $m / z\left[\mathrm{M}+\mathrm{H}^{+}\right]$calcd for $\mathrm{C}_{11} \mathrm{H}_{11} \mathrm{~N}_{2} \mathrm{O}_{2}$ : 203.0821; found: 203.0832 .

\section{4-Formyl-2-(3-methoxyphenyl)-1H-imidazole (5a)}

White solid; yield: $19.2 \mathrm{mg}(93 \%) ; \mathrm{mp} 172-173^{\circ} \mathrm{C}$.

IR (thin film): 3436, 3025, 1565, 1502, 843, 752, $723 \mathrm{~cm}^{-1}$.

${ }^{1} \mathrm{H}$ NMR (400 MHz, CD $\left.{ }_{3} \mathrm{OD}\right): \delta=9.69(\mathrm{~s}, 1 \mathrm{H}), 7.96(\mathrm{~s}, 1 \mathrm{H}), 7.60(\mathrm{~s}, 1$ $\mathrm{H}), 7.57(\mathrm{~d}, J=8.0 \mathrm{~Hz}, 1 \mathrm{H}), 7.32(\mathrm{t}, J=8.0 \mathrm{~Hz}, 1 \mathrm{H}), 6.93\left(\mathrm{dd}, J_{1}=8.0 \mathrm{~Hz}\right.$, $\left.J_{2}=2.4 \mathrm{~Hz}, 1 \mathrm{H}\right)$.

${ }^{13} \mathrm{C}$ NMR $\left(100 \mathrm{MHz}, \mathrm{CD}_{3} \mathrm{OD}\right): \delta=184.3,161.5,154.3,141.5,136.6$, $133.4,130.9,119.6,116.4,112.4,55.8$.

MS (ESI): $m / z=203.2\left[\mathrm{M}+\mathrm{H}^{+}\right]$.

HRMS (ESI): $m / z\left[\mathrm{M}+\mathrm{H}^{+}\right]$calcd for $\mathrm{C}_{11} \mathrm{H}_{11} \mathrm{~N}_{2} \mathrm{O}_{2}$ : 203.0821; found: 203.0838 .

\section{4-Formyl-2-[4-(trifluoromethoxy)phenyl]-1H-imidazole (6a)} White solid; yield: $20.9 \mathrm{mg}$ (85\%); $\mathrm{mp} 171-172{ }^{\circ} \mathrm{C}$.

IR (thin film): 3424, 3112, 1448, 822, 746, $720 \mathrm{~cm}^{-1}$.

${ }^{1} \mathrm{H}$ NMR (400 MHz, CD $\mathrm{OD}$ ): $\delta=14.38$ (s, $\left.1 \mathrm{H}\right), 10.59$ (s, $\left.1 \mathrm{H}\right), 8.99(\mathrm{br}$ $\mathrm{s}, 3 \mathrm{H}), 8.31(\mathrm{~d}, J=8.0 \mathrm{~Hz}, 1 \mathrm{H})$.

${ }^{13} \mathrm{C}$ NMR $\left(100 \mathrm{MHz}, \mathrm{CD}_{3} \mathrm{OD}\right): \delta=187.3,151.6,131.3,130.5,130.5$, $126.5,124.1,124.1,124.0,121.4,118.9$.

MS (ESI): $m / z=257.2\left[\mathrm{M}+\mathrm{H}^{+}\right]$.

HRMS (ESI): $m / z\left[\mathrm{M}+\mathrm{H}^{+}\right]$calcd for $\mathrm{C}_{11} \mathrm{H}_{8} \mathrm{~F}_{3} \mathrm{~N}_{2} \mathrm{O}_{2}$ : 257.0538; found: 257.0544 .

\section{4-Formyl-2-[4-(trifluoromethyl)phenyl]-1H-imidazole (7a)}

White solid; yield: $20.1 \mathrm{mg}$ (84\%); $\mathrm{mp} 168-169{ }^{\circ} \mathrm{C}$.

IR (thin film): 3411, 1552, 1418, 1140, 1092, $750 \mathrm{~cm}^{-1}$.

${ }^{1} \mathrm{H}$ NMR $\left(400 \mathrm{MHz}, \mathrm{CD}_{3} \mathrm{OD}\right): \delta=10.61(\mathrm{~s}, 1 \mathrm{H}), 9.07(\mathrm{~d}, J=8.0 \mathrm{~Hz}, 2 \mathrm{H})$, $8.99(\mathrm{~s}, 1 \mathrm{H}), 8.67(\mathrm{~d}, J=8.0 \mathrm{~Hz}, 2 \mathrm{H})$.

${ }^{13} \mathrm{C}$ NMR (100 MHz, $\left.\mathrm{CD}_{3} \mathrm{OD}\right): \delta=186.1,142.4,136.0,135.2,131.9$ (q), 129.0, 129.0, $128.5(\mathrm{q}), 128.5$ (q), 128.1, 125.4 .

MS (ESI): $m / z=241.2\left[\mathrm{M}+\mathrm{H}^{+}\right]$.

HRMS (ESI): $m / z\left[\mathrm{M}+\mathrm{H}^{+}\right]$calcd for $\mathrm{C}_{11} \mathrm{H}_{8} \mathrm{~F}_{3} \mathrm{~N}_{2} \mathrm{O}$ : 241.0589; found: 241.0598 .

2-(3,5-Difluorophenyl)-4-formyl-1H-imidazole (8a)

White solid; yield: $16.2 \mathrm{mg}$ (78\%); $\mathrm{mp} 152-153^{\circ} \mathrm{C}$. 
IR (thin film): 3502, 1548, 1420, 1088, $742 \mathrm{~cm}^{-1}$.

${ }^{1} \mathrm{H}$ NMR (400 MHz, CD $\left.{ }_{3} \mathrm{OD}\right): \delta=9.80(\mathrm{~s}, 1 \mathrm{H}), 8.02(\mathrm{~s}, 1 \mathrm{H}), 7.60(\mathrm{~s}, 1$ $\mathrm{H}), 7.59(\mathrm{~s}, 1 \mathrm{H}), 7.06(\mathrm{~s}, 1 \mathrm{H})$.

${ }^{13} \mathrm{C}$ NMR (100 MHz, $\left.\mathrm{CD}_{3} \mathrm{OD}\right): \delta=184.2,166.1,163.6,149.0,140.8$, $133.6(\mathrm{t}), 133.2,110.2(\mathrm{q}), 109.2(\mathrm{~d}), 105.9(\mathrm{t})$.

MS (ESI): $m / z=209.2\left[\mathrm{M}+\mathrm{H}^{+}\right]$.

HRMS (ESI): $m / z\left[\mathrm{M}+\mathrm{H}^{+}\right]$calcd for $\mathrm{C}_{10} \mathrm{H}_{7} \mathrm{~F}_{2} \mathrm{~N}_{2} \mathrm{O}$ : 209.0526; found: 209.0533.

\section{2-(2,5-Difluorophenyl)-4-formyl-1H-imidazole (9a)}

White solid; yield: $16.4 \mathrm{mg}$ (79\%); $\mathrm{mp} 150-151^{\circ} \mathrm{C}$.

IR (thin film): 3498, 1546, 1418, 1088, 866, $740 \mathrm{~cm}^{-1}$.

${ }^{1} \mathrm{H}$ NMR $\left(400 \mathrm{MHz}, \mathrm{CDCl}_{3}\right): \delta=9.77(\mathrm{~s}, 1 \mathrm{H}), 7.97(\mathrm{~m}, 1 \mathrm{H}), 7.83(\mathrm{~s}, 1$ $\mathrm{H}), 7.07$ ( $\mathrm{m}, 2 \mathrm{H})$.

${ }^{13} \mathrm{C}$ NMR $\left(100 \mathrm{MHz}, \mathrm{CDCl}_{3}\right): \delta=177.5,159.2,156.8,156.0,153.8$, $139.1,117.5,116.6,116.4,114.7$.

MS (ESI): $m / z=209.2\left[\mathrm{M}+\mathrm{H}^{+}\right]$

HRMS (ESI): $m / z\left[\mathrm{M}+\mathrm{H}^{+}\right]$calcd for $\mathrm{C}_{10} \mathrm{H}_{7} \mathrm{~F}_{2} \mathrm{~N}_{2} \mathrm{O}$ : 209.0526; found: 209.0538 .

\section{2-(2,3-Difluorophenyl)-4-formyl-1H-imidazole (10a) $)^{17}$}

White solid; yield: $16.6 \mathrm{mg}$ (80\%); mp $159-160{ }^{\circ} \mathrm{C}$.

${ }^{1} \mathrm{H}$ NMR $\left(400 \mathrm{MHz}, \mathrm{CD}_{3} \mathrm{OD}\right): \delta=9.72(\mathrm{~s}, 1 \mathrm{H}), 7.95(\mathrm{~s}, 1 \mathrm{H}), 7.69(\mathrm{t}, J=$ $8.4 \mathrm{~Hz}, 1 \mathrm{H}), 7.29(\mathrm{q}, J=8.4 \mathrm{~Hz}, 1 \mathrm{H}), 7.20(\mathrm{~m}, 1 \mathrm{H})$.

${ }^{13} \mathrm{C}$ NMR (100 MHz, CD ${ }_{3} \mathrm{OD}$ ): $\delta=184.8,153.4$ (d), 150.8 (q), 148.1 (d), $145.1,141.3,131.7,126.3$ (q), 125.7, 119.6 (d).

HRMS (ESI): $m / z\left[\mathrm{M}+\mathrm{H}^{+}\right]$calcd for $\mathrm{C}_{10} \mathrm{H}_{7} \mathrm{~F}_{2} \mathrm{~N}_{2} \mathrm{O}$ : 209.0526; found: 209.0544.

\section{4-Formyl-2-(pyridin-3-yl)-1H-imidazole (11a)}

White solid; yield: $16.1 \mathrm{mg}$ (93\%); $\mathrm{mp} 135-136{ }^{\circ} \mathrm{C}$.

IR (thin film): 1575, 1451, 1429, 1023, 843, 786, 752, $697 \mathrm{~cm}^{-1}$.

${ }^{1} \mathrm{H}$ NMR (400 MHz, CD $\mathrm{OD}$ ): $\delta=9.54(\mathrm{~s}, 1 \mathrm{H}), 9.10(\mathrm{~s}, 1 \mathrm{H}), 8.34$ (dd, $\left.J_{1}=4.8 \mathrm{~Hz}, J_{2}=1.2 \mathrm{~Hz}, 1 \mathrm{H}\right), 8.31\left(\mathrm{dd}, J_{1}=8.0 \mathrm{~Hz}, J_{2}=1.2 \mathrm{~Hz}, 1 \mathrm{H}\right), 7.85$ $(\mathrm{s}, 1 \mathrm{H}), 7.35$ (dd, $J_{1}=8.0 \mathrm{~Hz}, J_{2}=4.8 \mathrm{~Hz}, 1 \mathrm{H}$ ).

${ }^{13} \mathrm{C}$ NMR $\left(100 \mathrm{MHz}, \mathrm{CD}_{3} \mathrm{OD}\right): \delta=184.7,154.3,148.9,147.9,143.6$, $140.4,135.3,131.5,125.2$.

MS (ESI): $m / z=174.2\left[\mathrm{M}+\mathrm{H}^{+}\right]$.

HRMS (ESI): $m / z\left[\mathrm{M}+\mathrm{H}^{+}\right]$calcd for $\mathrm{C}_{9} \mathrm{H}_{8} \mathrm{~N}_{3} \mathrm{O}$ : 174.0667; found: 174.0679.

\section{4-Formyl-2-isobutyl-1H-imidazole (12a)}

White powder; yield: $10.0 \mathrm{mg}$ (65\%); $\mathrm{mp} 110-112{ }^{\circ} \mathrm{C}$.

IR (thin film): 3008, 1481, 1428, 1411, 1143, 1006, $727 \mathrm{~cm}^{-1}$.

${ }^{1} \mathrm{H}$ NMR (400 MHz, $\left.\mathrm{CD}_{3} \mathrm{OD}\right): \delta=10.42(\mathrm{~s}, 1 \mathrm{H}), 8.67$ (s, $\left.1 \mathrm{H}\right), 3.32(\mathrm{~d}, J=$ $7.6 \mathrm{~Hz}, 2 \mathrm{H}), 2.85(\mathrm{~m}, 1 \mathrm{H}), 1.68(\mathrm{~d}, J=7.2 \mathrm{~Hz}, 6 \mathrm{H})$.

${ }^{13} \mathrm{C}$ NMR $\left(100 \mathrm{MHz}, \mathrm{CD}_{3} \mathrm{OD}\right): \delta=184.7,154.7,135.6,127.7,39.4,30.3$, 24.8.

MS (ESI): $m / z=153.2\left[\mathrm{M}+\mathrm{H}^{+}\right]$.

HRMS (ESI): $m / z\left[\mathrm{M}+\mathrm{H}^{+}\right]$calcd for $\mathrm{C}_{8} \mathrm{H}_{13} \mathrm{~N}_{2} \mathrm{O}$ : 153.1028; found: 153.1038 .

\section{Acknowledgment}

This research was supported by the NSFC (No. 81001383) and the Doctoral Foundation of the Ministry of Education of China (No. 20105103120009).

\section{Supporting Information}

Supporting information for this article is available online at http://dx.doi.org/10.1055/s-0034-1379201.

\section{References}

(1) Luca, L. D. Curr. Med. Chem. 2006, 13, 1.

(2) Rani, N.; Sharma, A.; Singh, R. Mini-Rev. Med. Chem. 2013, 13, 1812.

(3) Jin, Z. Nat. Prod. Rep. 2013, 30, 869.

(4) Mombelli, P.; Le Chapelain, C.; Munzinger, N.; Joliat, E.; Illarionov, B.; Schweizer, W. B.; Hirsch, A. K. H.; Fischer, M.; Bacher, A.; Diederich, F. Eur. J. Org. Chem. 2013, 1068.

(5) Assadieskandar, A.; Amini, M.; Ostad, S. N.; Riazi, G. H.; Cheraghi-Shavi, T.; Shafiei, B.; Shafiee, A. Bioorg. Med. Chem. 2013, 21, 2703.

(6) Assadieskandar, A.; Amirhamzeh, A.; Salehi, M.; Ozadali, K.; Ostad, S. N.; Shafiee, A.; Amini, M. Bioorg. Med. Chem. 2013, 21, 2355.

(7) (a) Wu, Y.; Zhou, X.-P.; Yang, J.-R.; Li, D. Chem. Commun. 2013, 49, 3413. (b) Murase, M.; Yamauchi, S.; Sakamoto, S.; Takahashi, S.; Matsumoto, N.; Tsuchimoto, M. Polyhedron 2013, 59, 76. (c) Furushou, D.; Hashibe, T.; Fujinami, T.; Nishi, K.; Hagiwara, H.; Matsumoto, N.; Sunatsuki, Y.; Kojima, M.; Iijima, S. Polyhedron 2013, 52, 1489. (d) Sívek, R.; Bureš, F.; Pytela, O.; Kulhánek, J. Molecules 2008, 13, 2326.

(8) Hu, B.; Wang, Z.; Ai, N.; Zheng, J.; Liu, X.-H.; Shan, S.; Wang, Z. Org. Lett. 2011, 13, 6362.

(9) (a) Tang, D.; Wu, P.; Liu, X.; Chen, Y.-X.; Guo, S.-B.; Chen, W.-L.; Li, J.-G.; Chen, B.-H. J. Org. Chem. 2013, 78, 2746. (b) MaGee, D. I.; Bahramnejad, M.; Dabiri, M. Tetrahedron Lett. 2013, 54, 2591. (c) Li, S.; Li, Z.; Yuan, Y.; Li, Y.; Zhang, L.; Wu, Y. Chem. Eur. J. 2013, 19, 1496. (d) Li, J.; Neuville, L. Org. Lett. 2013, 15, 1752.

(10) (a) Dhainaut, A.; Tizot, A.; Raimbaud, E.; Lockhart, B.; Lestage, P.; Goldstein, S. J. Med. Chem. 2000, 43, 2165. (b) Griffiths, G. J.; Hauck, M. B.; Imwinkelried, R.; Kohr, J.; Roten, C. A.; Stucky, G. C.; Gosteli, J. J. Org. Chem. 1999, 64, 8084. (c) Ando, N.; Terashima, S. Tetrahedron 2010, 66, 6224.

(11) (a) Sunatsuki, Y.; Kawamoto, R.; Fujita, K.; Maruyama, H.; Suzuki, T.; Ishida, H.; Kojima, M.; Iijima, S.; Matsumoto, N. Coord. Chem. Rev. 2010, 254, 1871. (b) Itoh, K.; Hayashi, H.; Furutachi, H.; Matsumoto, T.; Nagatomo, S.; Tosha, T.; Terada, S.; Fujinami, S.; Suzuki, M.; Kitagawa, T. S. J. Am. Chem. Soc. 2005 , $127,5212$.

(12) Advances in Heterocyclic Chemistry; Vol. 111; Katritzky, A. R., Ed.; Academic Press: San Diego, 2014.

(13) Sun, H. B.; Zheng, G. J.; Wang, Y. P.; Wang, X. J.; Xiang, W. S. Chin. Chem. Lett. 2009, 20, 269.

(14) Reiter, L. A. J. Org. Chem. 1987, 52, 2714.

(15) (a) Zen, S.; Harada, K.; Nakamura, H.; Iitaka, Y. Bull. Chem. Soc. Jpn. 1988, 61, 2881. (b) Anderson, M.; Andrews, D. M.; Barker, A. J.; Brassington, C. A.; Breed, J.; Byth, K. F.; Culshaw, J. D.; Finlay, M. R. V.; Fisher, E.; McMiken, H. H. J.; Green, C. P.; Heaton, D. W.; Nash, I. A.; Newcombe, N. J.; Oakes, S. E.; Pauptit, R. A.; Roberts, 
A.; Stanway, J. J.; Thomas, A. P.; Tucker, J. A.; Walker, M.; Weir, H. M. Bioorg. Med. Chem. Lett. 2008, 18, 5487. (c) Thompson, S. K.; Murthy, K. H. M.; Zhao, B.; Winborne, E.; Green, D. W.; Fisher, S. M.; DesJarlais, R. L.; Tomaszek, T. A. Jr.; Meek, T. D. J. Med. Chem. 1994, 37, 3100. (d) Anderson, L.; Arzel, E.; Berg, S.; Burrows, J.; Hellberg, S.; Huerta, F.; Pedersen, T.; Rein, T.;
Rotticci, D.; Staaf, K.; Turek, D. (AstraZeneca AB) WO 2007/40440, 2007. (e) Burrows, J.; Huerta, F.; Rein, T.; Rotticci, D.; Staaf, K.; Turek, D. (AstraZeneca AB) WO 2008/2245, 2008.

(16) (a) Gao, F.; Wang, D.; Huang, X. Fitoterapia 2013, 90, 79. (b) Chen, X.-X.; Gao, F.; Wang, Q.; Huang, X.; Wang, D. Fitoterapia 2014, 92, 111.

(17) Haruyuki, N.; Yasuyoshi, A.; Keizo, H. (Takeda Pharmaceutical Company Limited) US 2009/156642 A1, 2009.

This article differs from the e-first online version only in its layout; no content has been changed. 